\title{
Prevalence of Asymptomatic Bacteriuria, Associated Factors and Antimicrobial Susceptibility Profile of Bacteria Among Pregnant Women Attending Saint Paul's Hospital Millennium Medical College, Addis Ababa, Ethiopia
}

This article was published in the following Dove Press journal:

Therapeutics and Clinical Risk Management

\author{
Yasin Awol Wabe $\mathbb{D}^{\prime}$ \\ Dawit Yihdego Reda ${ }^{2}$ \\ Estifanos Tsige Abreham (1D ${ }^{3}$ \\ Degeuf Beyene Gobene ${ }^{3}$ \\ Musa Mohammed $\mathrm{Ali}^{2}$ \\ 'Werabe Comprehensive Specialized \\ Hospital, Worabe, Ethiopia; ${ }^{2} \mathrm{Hawassa}$ \\ University, School of Medical Laboratory \\ Science, Hawassa, Ethiopia; ${ }^{3}$ Ethiopian \\ Public Health Institute, Addis Ababa, \\ Ethiopia
}

Introduction: Asymptomatic bacteriuria (ASB) is the presence of bacteria in significant quantity in the absence of signs and symptoms of urinary tract infection (UTI). ASB, if it occurs during pregnancy, can cause serious complications both among fetus and pregnant women.

Objective: The aim of this study was to determine the prevalence of ASB, its associated factors, and antimicrobial susceptibility profile of bacterial isolates among pregnant women. Methods: A cross-sectional study was conducted from July to September 2019 among 290 pregnant women at Saint Paul's Hospital Millennium Medical College, Addis Ababa, Ethiopia. Clean-catch midstream urine specimens were collected using sterile containers and cultured on MacConkey agar and sheep blood agar to isolate bacteria. Socio-demographic and obstetric data were collected using a structured questionnaire. Data were analyzed by SPSS version 22. The association between ASB and risk factors was assessed using logistic regressions. A $p$-value $\leq 0.05$ was considered as a cut point to determine the significant association.

Results: From 290 study participants, $16.9 \%$ with 95 CI [13.1, 21.5] were positive for ASB. The predominant bacteria were Escherichia coli (43\%) and Staphylococcus aureus (20\%). Majority of E. coli $(91.0 \%)$ were susceptible to nitrofurantoin and gentamycin; most of them were resistant to amoxicillin (86.4\%) and cotrimoxazole $(77.7 \%)$. The proportion of multidrug resistance (MDR) isolates was $57.1 \%$. Previous infection with UTI, previous history of catheterization, and natural abortion were significantly associated with ASB.

Conclusion: In the study area, ASB is prevalent in the study area indicating the importance of screening of ASB and possible treatment to prevent its consequences.

Keywords: asymptomatic bacteriuria, antibiotic susceptibility, pregnant woman, Addis Ababa, Ethiopia

\section{Introduction}

Urinary tract infection (UTI) is an infection of the urinary tract system; it can occur both in the community and hospital environment. ${ }^{1,2}$ Even though all age groups are affected by UTI, pregnant women, ${ }^{3-5}$ and immuno-compromised individuals ${ }^{6}$ are more susceptible. Generally, women are at greater risk of UTI as compared to males because of anatomical position and short urethra. ${ }^{6,7}$ If UTI occurs among pregnant women; it can be with symptoms or without symptoms. Urinary tract infection without signs and symptoms is known as asymptomatic bacteriuria (ASB).
Correspondence: Yasin Awol Wabe Email awolyasin30@gmail.com
Therapeutics and Clinical Risk Management 2020:16 923-932 
Asymptomatic bacteriuria is the presence of a significant quantity of bacteria $\left(\geq 10^{5}\right)$ in a urine specimen in the absence of signs and symptoms of UTI. ${ }^{8}$ Asymptomatic bacteriuria may progress to symptomatic UTI during pregnancy because of the following reasons: altered hormones, physiological changes, and reduced immunity. ${ }^{9}$ Other factors that could predispose pregnant women to bacteriuria are age, lower socioeconomic status, multiparty, past history of UTI, history of catheterization, aminoaciduria, anemia, and diabetes mellitus. ${ }^{10-12}$

In most cases, the leading cause of UTI is E. coli, ${ }^{13}$ while other Gram-negative bacteria such as Pseudomonas aeruginosa, Klebsiella pneumoniae, Proteus species, and some Gram-positive bacteria are also responsible. ${ }^{14}$ Significant numbers of pregnant women with ASB may develop acute upper UTI. ${ }^{15}$ Upper UTI can have a direct and indirect impact on the fetus. ${ }^{16,17}$

There are scarce data on the prevalence of ASB in Addis Ababa. The aim of this study was to determine the prevalence of ASB, its associated factors, and antimicrobial susceptibility profile of bacterial isolates among pregnant women attending Saint Paul's Hospital Millennium Medical College (SPHMMC) Addis Ababa, Ethiopia.

\section{Methods}

\section{Study Area}

This study was conducted at SPHMMC which is located in Addis Ababa, the capital city of Ethiopia. The hospital provides health care services for about two million patients per year. Every month, about 830 pregnant women visit antenatal care unit of the hospital for follow-up.

\section{Study Design and Study Population}

A hospital-based cross-sectional study was conducted from July to September 2019. Pregnant women of all gestational age without signs and symptoms of UTI (such as dysuria, haematuria, fever, suprapubic pain, flank pain, the urgency of urination) were included in the study. Pregnant women with a history of antibiotics in the last 15 days and those with known congenital anomalies of the urinary tract system were excluded from the study.

\section{Variables of the Study \\ Dependent Variables}

Prevalence of ASB.

\section{Independent Variables}

Socio-demographic factors and other related factors such as the history of UTI, hemoglobin level, history of catheterization, educational status, monthly income, gestational age, history of natural abortion, parity, history of stillbirth, etc.

\section{Sample Size Determination}

The sample size was determined using a single population proportion formula, by considering the prevalence of UTI $(21.2 \%)$ reported from Tigray Regional State, Ethiopia, ${ }^{4}$ $95 \%$ confidence interval and a $5 \%$ margin of error. The total sample size after considering risk factors and $10 \%$ contingency was equal to 290 .

\section{Sampling Technique}

To recruit study participants systematic random sampling technique was used by considering the three months of study period. According to the hospital plan and the past three months' performance report, about 1518 pregnant women were expected to visit the antenatal care unit during the study period. This estimate was divided into the sample size $(\mathrm{n}=290)$ to determine the sampling interval $(\mathrm{K}$ value) which was five. The first study participant was selected by lottery method; then onward, every 5 th pregnant women were invited to participate in the study until the required sample size was obtained.

\section{Data Collection}

Socio-demographic and factors that could predispose to ASB (history of UTI, hemoglobin level, history of catheterization, educational status, monthly income, gestational age, history of natural abortion, parity, history of stillbirth, etc.) were captured by using a structured questionnaire. Participants were instructed on how to collect clean-catch midstream urine. They were informed to clean their hands with water and soap then cleanse their periurethral area with a sterile cotton swab soaked in normal saline to reduce contamination. About 10-15 milliliter of clean-catch midstream urine was collected from all study participants using a widemouthed sterile container. Specimens were transported to the microbiology laboratory of Ethiopian Public Health Institute (EPHI) within 1-2 hours after collection. Uncentrifuged, well mixed, urine of $0.001 \mathrm{~mL}$ was inoculated onto MacConkey agar and 5\% sheep blood agar by a calibrated wire loop and then incubated aerobically at $37^{\circ} \mathrm{C}$ overnight. Colony count per $\mathrm{mL}$ of the sample was 
multiplied by 1000 to get the total colony-forming unit (CFU) per $\mathrm{mL}$ of urine. A plate with $10^{5} \mathrm{CFU} / \mathrm{mL}$ colony count or greater was considered as significant growth. ${ }^{18}$ Bacteria were identified by colony characteristics, Gram reaction, catalase test, coagulase test, and biochemical tests. $^{19}$

\section{Antimicrobial Susceptibility Testing}

Antimicrobial susceptibility testing was performed using disk diffusion method on Mueller-Hinton agar (Oxoid Ltd, Hampshire, UK) according to Clinical and Laboratory Standards Institute (CLSI) guideline. ${ }^{20}$ Antimicrobials tested were comprised of ampicillin $(10 \mu \mathrm{g})$, amoxicillin $(20 \mu \mathrm{g})$, amoxicillin-clavulanic acid $(20 / 10 \mu \mathrm{g})$, ciprofloxacin $(5 \mu \mathrm{g})$, ceftriaxone $(30 \mu \mathrm{g})$, azithromycin $(15 \mu \mathrm{g})$ cefuroxime $(30 \mu \mathrm{g})$, nitrofurantoin $(300 \mu \mathrm{g})$, erythromycin $(15 \mu \mathrm{g})$, cotrimoxazole $(25 \mu \mathrm{g})$, tetracycline $(30 \mu \mathrm{g})$, clindamycin $(2 \mu \mathrm{g})$, gentamicin $(10 \mu \mathrm{g})$, penicillin $(30 \mu \mathrm{g})$, and meropenem $(10 \mu \mathrm{g})$.

\section{Quality Control}

To maintain the quality of socio-demographic and obstetric data, the questionnaire was pretested among pregnant women that represent $5 \%$ of the total sample size at Tikur Anbesa Specialized Hospital. Originally the questionnaire was prepared in English then it was translated to Amharic and finally translated back to English by different translators to check its consistency. The sterility of all culture media was checked by incubating $5 \%$ of them without inoculation at $37{ }^{\mathrm{O}} \mathrm{C}$. The performance of culture media was checked by using control strains such as E. coli (ATCC-25,922), S. aureus (ATCC-25,923), and P. aeruginosa (ATCC-27,853) throughout the study.

\section{Operational Definitions}

\section{Asymptomatic Bacteriuria (ASB)}

Bacterial colony count greater than or equal to $10^{5} \mathrm{cfu} / \mathrm{mL}$ from urine sample collected from a person with no signs and symptoms of UTIs (such as dysuria, haematuria, fever, suprapubic pain, flank pain, the urgency of urination). ${ }^{21}$

\section{Multidrug-Resistance (MDR)}

Bacteria that show resistance to two or more classes of antimicrobial agents. $^{22}$

\section{Data Processing and Analysis}

Data were cleaned, coded and entered using Epi Info Version 7.2 and analyzed by SPSS software version 22. Descriptive statistics was used to determine the prevalence of ASB, the proportion of bacteria, and AST profiles of bacteria. To determine factors associated with ASB bivariate and multivariable logistic regression were used. Variables with a $p$-value less than 0.25 in bivariate analysis were selected for further analysis by multivariable logistic regression. A $p$-value of less than 0.05 was taken as a cut point to determine significant association.

\section{Results}

\section{Socio-Demographic Characteristics of Study Participants}

In this study, a total of 290 pregnant women were included with a response rate of $100 \%$. The mean age of the participants was $27 \pm 4.5$ years. Two hundred-eighty (96.6\%) participants were married. Twenty percent of participants attended the College or University level of education. More than half of the study participants resided in an urban area (Table 1).

\section{Prevalence of Asymptomatic Bacteriuria}

Out of 290 pregnant women without signs and symptoms of UTI, 49 (16.9\%) $(95 \%, \mathrm{CI}=13.1-21.5)$ had significant bacteriuria. Among participants with significant bacteriuria, $47(95.9 \%)$ were positive for one bacteria while 2 (4.1\%) were positive for more than one organism. In this

Table I Socio-Demographic Characteristics of Pregnant Women Attending the Antenatal Clinic of the Saint Paul's Hospital Millennium Medical College, Addis Ababa, Ethiopia, 2019 (N= 290)

\begin{tabular}{|c|c|c|c|}
\hline Variables & Category & Frequency & Percent \\
\hline $\begin{array}{l}\text { Age in } \\
\text { years }\end{array}$ & $\begin{array}{l}18-25 \\
26-34 \\
35-43\end{array}$ & $\begin{array}{l}101 \\
169 \\
20\end{array}$ & $\begin{array}{l}34.8 \\
58.3 \\
6.9\end{array}$ \\
\hline Occupation & $\begin{array}{l}\text { Housewife } \\
\text { Merchant } \\
\text { Government employed } \\
\text { Student }\end{array}$ & $\begin{array}{l}193 \\
55 \\
38 \\
4\end{array}$ & $\begin{array}{l}66.6 \\
19.0 \\
13.0 \\
1.4\end{array}$ \\
\hline $\begin{array}{l}\text { Marital } \\
\text { status }\end{array}$ & $\begin{array}{l}\text { Married } \\
\text { Single }\end{array}$ & $\begin{array}{l}280 \\
10\end{array}$ & $\begin{array}{l}96.6 \\
3.4\end{array}$ \\
\hline Education & $\begin{array}{l}\text { No formal education } \\
\text { Primary school } \\
\text { Secondary school } \\
\text { College or University level } \\
\text { education }\end{array}$ & $\begin{array}{l}26 \\
125 \\
81 \\
58\end{array}$ & $\begin{array}{l}3.4 \\
43.1 \\
27.9 \\
20.0\end{array}$ \\
\hline Residence & $\begin{array}{l}\text { Urban } \\
\text { Rural }\end{array}$ & $\begin{array}{l}249 \\
41\end{array}$ & $\begin{array}{l}85.9 \\
14.1\end{array}$ \\
\hline
\end{tabular}


study, eight different types of bacteria were identified. Out of 49 bacteria isolated, 29 (59.2\%) were Gram-negative bacteria and $20(40.8 \%)$ were Gram-positive bacteria. The predominant bacteria were E. coli $21(43 \%)$ and $S$. aureus 10 (20.4\%) followed by S. saprophyticus 7(14.3\%), K. Pneumoniae 3(6.1\%), Enterobacter cloacae 2(4.1\%), Enterococcus faecalis 2(4.1\%), Klebsiella oxytoca 1 (2.1\%), and Enterobacter aerogens 1(2.1\%). The two mixed cultures contained E. coli with Candida albicans and $S$. saprophyticus with C. albicans.

\section{Factors Associated with Asymptomatic Bacteriuria}

High prevalence of ASB was observed among participants within the age group of $26-34$ years $(17.8 \%)$, housewives (18.1\%), urban residents (17.7\%), married (17.1\%), and with no formal education (23.1\%). However, there was no significant association between ASB and sociodemographic characteristics (Table 2). In multivariable analysis, the prevalence of ASB was significantly associated with a history of UTI $[\mathrm{AOR}=3.11,95 \% \mathrm{Cl}: 1.42$, 6.79], history catheterization $[\mathrm{AOR}=2.31,95 \% \mathrm{Cl}: 1.04$, 5.73], anemia [AOR $=10.49,95 \% \mathrm{Cl}: 4.54,24.04]$, and history of natural abortion $[\mathrm{AOR}=2.36,95 \% \mathrm{Cl}: 1.13$, 4.94] (Table 3).

\section{Antimicrobial Susceptibility Profile of Bacteria}

The antimicrobial susceptibility profile of Gram-negative bacteria (susceptible bacteria) falls within the range of $13.8 \%-93.1 \%$. The majority of the Gram-negative bacteria were susceptible to nitrofurantoin $(\mathrm{n}=27 ; 93.1 \%)$, gentamycin $(\mathrm{n}=25 ; 86.2 \%)$, ceftriaxone $(\mathrm{n}=24 ; 82.8 \%)$, cefuroxime $(\mathrm{n}=23 ; 82.8 \%)$, and meropenem $(\mathrm{n}=22 ; 75.9 \%)$. Most Gram-negative bacteria were resistant to amoxicillin $(\mathrm{n}=23 ; 79.3 \%)$, cotrimoxazole $(\mathrm{n}=19 ; 65.5 \%)$, and amoxicillin-clavulanic acid $(\mathrm{n}=11 ; 37.9 \%)$. Most of E. coli $(\mathrm{n}=19 ; 86.4 \%)$ were resistant to amoxicillin (Table 4).

All Gram-positive bacteria were susceptible to nitrofurantoin $(\mathrm{n}=20 ; 100 \%)$. Majority were susceptible to azithromycin $(\mathrm{n}=18 ; 90.0 \%)$, gentamicin, and ciprofloxacin (for each $\mathrm{n}=17 ; 85.0 \%)$ and clindamycin $(\mathrm{n}=16 ; 80.0 \%$ ). Most of $S$. aureus were susceptible to gentamicin, ciprofloxacin, and azithromycin ( $\mathrm{n}=9 ; 90.0 \%$ ); however, 70\% were resistant to penicillin. Seventy-five percent of S. saprophyticus were susceptible to gentamycin, ciprofloxacin, clindamycin, and azithromycin (Table 5). Out of
49 bacteria isolated in this study, 28 (57.1\%) were MDR; $15(51.7 \%)$ of this belongs to Gram-negative while 13 (65.0\%) belongs to Gram-positive bacteria (Table 6).

\section{Discussion}

The prevalence of ASB among pregnant women in the current study $(16.9 \%)$ is in line with a study conducted in Bangladesh $(16.5 \%)^{23}$ and Adama, Ethiopia (16.1\%). ${ }^{24}$ However, it is low compared to the prevalence of ASB reported from Iraq $(42.9 \%),{ }^{25}$ Nigeria $(37.1 \%),{ }^{26}$ Saudi Arabia (32.1\%), ${ }^{27}$ and Adigrat, Ethiopia (21.2\%). ${ }^{4}$ In addition, the finding of the current study is higher than the prevalence of UTI reported from Nigeria $(11 \%)^{28}$ and Bahir Dar, Ethiopia (11.5\%). ${ }^{29}$ The variation could be attributed to social habits and education levels of the study participants.

The proportion of Gram-negative bacteria (59.2\%) identified in the present study is higher than the proportion of Gram-positive bacteria (40.8\%). This finding is in line with studies conducted in Gondar, Ethiopia, ${ }^{30}$ Tanzania, ${ }^{31}$ and Nigeria. ${ }^{26}$ E. coli was found to be the most prevalent (43\%) bacteria isolated in this study. A similar finding was reported from Adama, Ethiopia $(37.3 \%),{ }^{24}$ Kenya $(38.8 \%),{ }^{32}$ and Tanzania $(64.2 \%) .{ }^{33}$ This could be due to virulence factors harbored by the bacteria to colonize the urinary epithelium and difficultly of maintaining personal hygiene during pregnancy. The second predominant bacteria were S. aureus (20.4\%) followed by S. saprophyticus (14.3\%). This finding is comparable with the study conducted in Iraq $(25.0 \%)^{25}$ and Kenya $(29.7 \%) .^{32}$ The proportion of S. saprophyticus (14.3\%) in the current study is comparable with report from Adigrat, Ethiopia (12.7\%). ${ }^{4}$ In contrast, a high proportion of $S$. saprophyticus was reported from Bahir Dar, Ethiopia $(48.2 \%)^{29}$ and Nigeria $(44.5 \%){ }^{3}$

In the current study, participants with the previous history of UTI had about 3 times chance of developing ASB ( $p=0.004)$. A similar finding was reported from Gondar, Ethiopia ${ }^{30}$ and Egypt. ${ }^{34}$ The possible explanation for this association could be due to the existence of antibiotic-resistant strains from the previous infection. The history of catheterization was also significantly associated with ASB $(p=0.04)$. This is in line with a study conducted in Dessie, Ethiopia. ${ }^{5}$ The reason for this could be due to the introduction of bacteria to the sterile site during catheterization. Frequent and long-term catheterization could create a favorable environment for bacterial colonization and growth as a biofilm. Pregnant women with a history of 
Table 2 ASB Across Different Socio-Demographic Characteristics Among Pregnant Women Attending Antenatal Clinic of the Saint Paul's Hospital Millennium Medical College, Addis Ababa, Ethiopia, 2019 (N=290)

\begin{tabular}{|c|c|c|c|c|c|c|}
\hline \multirow[t]{2}{*}{ Variables } & \multirow{2}{*}{$\begin{array}{l}\text { Tested } \\
\text { n (\%) }\end{array}$} & \multicolumn{2}{|l|}{ ASB } & \multirow[t]{2}{*}{ COR $(95 \% \mathrm{Cl})$} & \multirow{2}{*}{\multicolumn{2}{|c|}{ p-value }} \\
\hline & & $\begin{array}{l}\text { Positive } \\
\text { n (\%) }\end{array}$ & $\begin{array}{l}\text { Negative } \\
\text { n(\%) }\end{array}$ & & & \\
\hline \multicolumn{7}{|c|}{ Age (in years) } \\
\hline & $\begin{array}{l}18-25 \\
26-34 \\
35-43\end{array}$ & $\begin{array}{l}101(34.8) \\
169(58.3) \\
20(6.9)\end{array}$ & $\begin{array}{l}17(16.8) \\
30(17.8) \\
2(10.0)\end{array}$ & $\begin{array}{l}84(83.2) \\
139(82.2) \\
18(90.0)\end{array}$ & $\begin{array}{l}\text { I } \\
\text { I.06 }(0.55,2.05) \\
0.54(0.12,2.58)\end{array}$ & $\begin{array}{l}0.847 \\
0.449\end{array}$ \\
\hline \multicolumn{7}{|c|}{ Occupation } \\
\hline & $\begin{array}{l}\text { Housewife } \\
\text { Merchant } \\
\text { Government Employed } \\
\text { Student }\end{array}$ & $\begin{array}{l}193(66.6) \\
55(19.0) \\
38(13.0) \\
4(1.4)\end{array}$ & $\begin{array}{l}35(18.1) \\
8(14.5) \\
5(13.2) \\
I(25.0)\end{array}$ & $\begin{array}{l}158(81.9) \\
47(85.5) \\
33(86.8) \\
3(75.0)\end{array}$ & $\begin{array}{l}0.66(0.07,6.58) \\
0.5 I(0.05,5.54) \\
0.45(0.04,5.27) \\
I\end{array}$ & $\begin{array}{l}0.727 \\
0.581 \\
0.528\end{array}$ \\
\hline \multicolumn{7}{|c|}{ Marital status } \\
\hline & $\begin{array}{l}\text { Married } \\
\text { Single }\end{array}$ & $\begin{array}{l}280(96.6) \\
10(3.4)\end{array}$ & $\begin{array}{l}48(17.1) \\
I(10.0)\end{array}$ & $\begin{array}{l}232(82.9) \\
9(90.0)\end{array}$ & $\begin{array}{l}1.86(0.23,15.04) \\
1\end{array}$ & 0.560 \\
\hline \multicolumn{7}{|c|}{ Educational status } \\
\hline & $\begin{array}{l}\text { No formal education } \\
\text { Primary school } \\
\text { Secondary school } \\
\text { Higher education }\end{array}$ & $\begin{array}{l}26(9.0) \\
125(43.1) \\
81(27.9) \\
37(12.8)\end{array}$ & $\begin{array}{l}6(23.1) \\
23(18.4) \\
15(18.5) \\
4(10.8)\end{array}$ & $\begin{array}{l}20(76.9) \\
102(81.6) \\
66(81.5) \\
33(89.2)\end{array}$ & $\begin{array}{l}3.18(0.87,11.59) \\
2.39(0.86,6.64) \\
2.41(0.82,7.05) \\
I\end{array}$ & $\begin{array}{l}0.08 \\
0.95 \\
0.11\end{array}$ \\
\hline \multicolumn{7}{|c|}{ Place of residence } \\
\hline & $\begin{array}{l}\text { Urban } \\
\text { Rural }\end{array}$ & $\begin{array}{l}249(85.9) \\
4 I(14.1)\end{array}$ & $\begin{array}{l}44(17.7) \\
5(12.2)\end{array}$ & $\begin{array}{l}205(82.4) \\
36(87.8)\end{array}$ & $\begin{array}{l}\text { I } \\
0.65(0.240-1.742)\end{array}$ & 0.389 \\
\hline \multicolumn{7}{|c|}{ Gestational age } \\
\hline & $\begin{array}{l}\text { Ist Trimester } \\
\text { 2nd Trimester } \\
\text { 3rd Trimester }\end{array}$ & $\begin{array}{l}51(17.6) \\
66(22.8) \\
173(59.6)\end{array}$ & $\begin{array}{l}8(15.7) \\
10(15.2) \\
31(17.9)\end{array}$ & $\begin{array}{l}43(84.3 \%) \\
56(84.8) \\
142(82.1)\end{array}$ & $\begin{array}{l}\text { I } \\
0.96(0.349,2.638) \\
I .173(0.502,2.742)\end{array}$ & $\begin{array}{l}0.937 \\
0.712\end{array}$ \\
\hline \multicolumn{7}{|l|}{ Parity } \\
\hline & $\begin{array}{l}\text { Nulliparous } \\
\text { Primiparous } \\
\text { Multiparous }\end{array}$ & $\begin{array}{l}86(29.7) \\
109(37.6) \\
95(32.8)\end{array}$ & $\begin{array}{l}17(19.8) \\
16(14.7) \\
16(16.8)\end{array}$ & $\begin{array}{l}69(80.2) \\
93(85.3) \\
79(83.2)\end{array}$ & $\begin{array}{l}\text { I } \\
0.69(0.33,1.47) \\
0.82(0.38,1.74)\end{array}$ & $\begin{array}{l}0.348 \\
0.611\end{array}$ \\
\hline \multicolumn{7}{|c|}{ History of still birth } \\
\hline & $\begin{array}{l}\text { Yes } \\
\text { No }\end{array}$ & $\begin{array}{l}14(4.5) \\
276(95.5)\end{array}$ & $\begin{array}{l}3(21.4) \\
46(16.7)\end{array}$ & $\begin{array}{l}\mathrm{II}(78.6) \\
230(83.3)\end{array}$ & $\begin{array}{l}1.36(0.17,2.50) \\
1\end{array}$ & 0.545 \\
\hline \multicolumn{7}{|c|}{ History of early neonatal death } \\
\hline & $\begin{array}{l}\text { Yes } \\
\text { No }\end{array}$ & $\begin{array}{l}14(4.8) \\
276(95.2)\end{array}$ & $\begin{array}{l}\text { I (7.1) } \\
48(17.4)\end{array}$ & $\begin{array}{l}13(92.9) \\
228(82.6)\end{array}$ & $\begin{array}{l}0.36(0.35,21.42) \\
I\end{array}$ & 0.338 \\
\hline \multicolumn{7}{|c|}{ History of diabetes } \\
\hline & $\begin{array}{l}\text { Yes } \\
\text { No }\end{array}$ & $\begin{array}{l}21(7.2) \\
269(92.8)\end{array}$ & $\begin{array}{l}5(23.8) \\
44(16.4)\end{array}$ & $\begin{array}{l}16(76.2) \\
225(83.6)\end{array}$ & $\begin{array}{l}1.59(0.22,1.79) \\
1\end{array}$ & 0.384 \\
\hline
\end{tabular}


Table 2 (Continued).

\begin{tabular}{|l|l|l|l|l|l|}
\hline Variables & $\begin{array}{l}\text { Tested } \\
\text { n (\%) }\end{array}$ & \multicolumn{2}{|l|}{ ASB } & \multirow{2}{*}{ COR (95\% Cl) } & \multirow{2}{*}{ p-value } \\
\cline { 3 - 4 } & $\begin{array}{l}\text { Positive } \\
\text { n (\%) }\end{array}$ & $\begin{array}{l}\text { Negative } \\
\text { n(\%) }\end{array}$ & \\
\hline
\end{tabular}

Abbreviations: COR, crude odds ratio; $\mathrm{Cl}$, confidence interval; ASB, asymptomatic bacteriuria.

Table 3 Bivariate and Multivariable Analyses of Factors Associated with ASB Among Pregnant Women Attending the Antenatal Clinic of the Saint Paul's Hospital Millennium Medical College, Addis Ababa, Ethiopia, 2019 ( $N=290)$

\begin{tabular}{|c|c|c|c|c|c|c|c|c|}
\hline \multirow[t]{2}{*}{ Variables } & \multirow{2}{*}{\multicolumn{2}{|c|}{ Tested n(\%) }} & \multicolumn{2}{|l|}{ ASB } & \multicolumn{2}{|c|}{ Bivariate Analysis } & \multicolumn{2}{|c|}{ Multivariate Analysis } \\
\hline & & & $\begin{array}{l}\text { Positive n } \\
\text { (\%) }\end{array}$ & $\begin{array}{l}\text { Negative n } \\
\text { (\%) }\end{array}$ & COR $(95 \% \mathrm{CI})$ & P-value & AOR $(95 \% \mathrm{Cl})$ & $p$-value \\
\hline \multicolumn{9}{|c|}{ History of urinary tract infection } \\
\hline & $\begin{array}{l}\text { Yes } \\
\text { No }\end{array}$ & $\begin{array}{l}91(31.4) \\
199(68.6)\end{array}$ & $\begin{array}{l}28(30.8) \\
21(10.6)\end{array}$ & $\begin{array}{l}63(69.2) \\
178(89.4)\end{array}$ & $\begin{array}{l}3.76(1.99,7.12) \\
1\end{array}$ & 0.001 & $3.11(1.42,6.79)$ & 0.004 \\
\hline \multicolumn{9}{|c|}{ Hemoglobin level } \\
\hline & $\begin{array}{l}<\mid \mathrm{lg} / \mathrm{dl} \text { (Anemic) } \\
\geq \mathrm{I} \mathrm{g} / \mathrm{dl} \text { (Normal) }\end{array}$ & $\begin{array}{l}33(11.4) \\
257(88.6)\end{array}$ & $\begin{array}{l}18(54.5) \\
31(12.0)\end{array}$ & $\begin{array}{l}15(45.5) \\
226(88.0)\end{array}$ & $\begin{array}{l}8.75(4.01,19.11) \\
1\end{array}$ & 0.0001 & $10.49(4.58,24.04)$ & 0.0001 \\
\hline \multicolumn{9}{|c|}{ History of catheterization } \\
\hline & $\begin{array}{l}\text { Yes } \\
\text { No }\end{array}$ & $\begin{array}{l}89(30.7) \\
201(69.3)\end{array}$ & $\begin{array}{l}23(25.8) \\
26(12.9)\end{array}$ & $\begin{array}{l}66(74.2) \\
175(87.1)\end{array}$ & $\begin{array}{l}2.34(1.25,4.39) \\
I\end{array}$ & 0.008 & $2.31(1.04,5.13)$ & 0.04 \\
\hline \multicolumn{9}{|c|}{ Educational status } \\
\hline & $\begin{array}{l}\text { No formal } \\
\text { education } \\
\text { Primary school } \\
\text { Secondary school } \\
\text { Higher education }\end{array}$ & $\begin{array}{l}26(9) \\
125(43.1) \\
8 \mid(27.9) \\
58(20.0)\end{array}$ & $\begin{array}{l}6(23.1) \\
23(18.4) \\
15(18.5) \\
5(8.6)\end{array}$ & $\begin{array}{l}20(76.9) \\
102(81.6) \\
66(81.5) \\
53(91.4)\end{array}$ & $\begin{array}{l}3.18(0.87, \\
\text { II.59) } \\
2.39(0.86,6.64) \\
2.14(0.82,7.05) \\
\text { I }\end{array}$ & $\begin{array}{l}0.08 \\
0.95 \\
0.11\end{array}$ & $\begin{array}{l}3.26(0.72-\mid 4.83) \\
2.04(0.63,6.62) \\
2.45(0.72,8.38)\end{array}$ & $\begin{array}{l}0.126 \\
0.233 \\
0.152\end{array}$ \\
\hline \multicolumn{9}{|c|}{ Average monthly income level (in Ethiopian Birr) } \\
\hline & $\begin{array}{l}\leq 500 \\
501-1000 \\
1001-1500 \\
1501-2000 \\
>2000\end{array}$ & $\begin{array}{l}20(6.9) \\
58(20.0) \\
29(10.0) \\
51(17.6) \\
132(45.5)\end{array}$ & $\begin{array}{l}3(15.0) \\
13(22.4) \\
3(10.3) \\
10(19.6) \\
20(15.2)\end{array}$ & $\begin{array}{l}17(85.0) \\
45(77.6) \\
26(89.7) \\
41(80.4) \\
I 12(84.8)\end{array}$ & $\begin{array}{l}0.98(0.26,3.68) \\
1.62(0.74,3.52) \\
0.64(0.18,2.34) \\
1.36(0.59,3.16) \\
\text { I }\end{array}$ & $\begin{array}{l}0.986 \\
0.226 \\
0.506 \\
0.466\end{array}$ & $\begin{array}{l}0.36(0.06,1.92) \\
1.17(0.46,2.95) \\
0.54(0.13,2.19) \\
0.75(0.26,2.12)\end{array}$ & $\begin{array}{l}0.232 \\
0.735 \\
0.390 \\
0.590\end{array}$ \\
\hline \multicolumn{9}{|c|}{ History of natural abortion } \\
\hline & $\begin{array}{l}\text { Yes } \\
\text { No }\end{array}$ & $\begin{array}{l}91(31.4) \\
199(68.6)\end{array}$ & $\begin{array}{l}24(26.4) \\
25(I 2.6)\end{array}$ & $\begin{array}{l}67(73.6) \\
174(87.4)\end{array}$ & $\begin{array}{l}2.49(1.33,4.66) \\
\text { I }\end{array}$ & 0.004 & $2.36(1.13,4.96)$ & 0.023 \\
\hline
\end{tabular}

Abbreviations: COR, crude odds ratio; $\mathrm{AOR}$, adjusted odds ratio; $\mathrm{Cl}$, confidence interval; $\mathrm{ASB}$, asymptomatic bacteriuria.

natural abortion were 2.36 times more likely to develop ASB $(p=0.023)$. The results of this study disagree with the finding reported from Cameron. ${ }^{35}$
Majority of Gram-negative bacteria isolated in the current study were susceptible to nitrofurantoin $(93.1 \%)$, gentamicin (85.2\%), ceftriaxone $(82.2 \%)$, cefuroxime $(79.3 \%)$, 
Table 4 Antimicrobial Susceptibility Profile of Gram-Negative Bacteria Isolated from Pregnant Women with ASB at Saint Paul's Hospital Millennium Medical College, Addis Ababa, Ethiopia, 2019 ( $n=29)$

\begin{tabular}{|c|c|c|c|c|c|c|c|c|c|c|c|c|}
\hline \multicolumn{2}{|c|}{ Type of Isolates (n) } & \multicolumn{11}{|c|}{ Antimicrobial Agents n (\%) } \\
\hline & & GEN & CIP & AMP & AMX & AMC & TET & NIT & CEF & CRX & MER & сOT \\
\hline \multirow[t]{3}{*}{ E. coli (22) } & $S$ & $20(91.0)$ & $18(81.8)$ & $10(45.5)$ & I(4.5) & $10(45.5)$ & $15(68.2)$ & $20(91.0)$ & $18(81.8)$ & $18(81.8)$ & $18(81.8)$ & $6(27.3)$ \\
\hline & I & $\mathrm{I}(4.5)$ & $\mathrm{I}(4.5)$ & - & $2(9.1)$ & $2(9.0)$ & - & - & - & $\mathrm{I}(4.5)$ & $3(13.7)$ & - \\
\hline & $\mathrm{R}$ & $\mathrm{I}(4.5)$ & $3(13.7)$ & $12(54.5)$ & $19(86.4)$ & $10(45.5)$ & $7(31.8)$ & $2(9.0)$ & $4(18.02)$ & $3(13.7)$ & $\mathrm{I}(4.5)$ & 16(77.7) \\
\hline \multirow[t]{3}{*}{ K. pneumoniae (3) } & $S$ & $\mathrm{I}(33.3)$ & $\mathrm{I}(33.3)$ & $2(66.7)$ & $\mathrm{I}(33.3)$ & $2(66.7)$ & $\mathrm{I}(33.3)$ & $3(100)$ & $\mathrm{I}(33.3)$ & $2(66.7)$ & $\mathrm{I}(33.3)$ & $\mathrm{I}(33.3)$ \\
\hline & I & $\mathrm{I}(33.3)$ & - & - & - & - & $\mathrm{I}(33.3)$ & - & I(33.3) & - & & - \\
\hline & $\mathrm{R}$ & $\mathrm{I}(33.4)$ & $2(66.7)$ & $\mathrm{I}(33.3)$ & $2(66.7)$ & $\mathrm{I}(33.3)$ & $\mathrm{I}(33.3)$ & - & $I(33.3)$ & $I(33.3)$ & $2(66.7)$ & $2(66.7)$ \\
\hline \multirow[t]{3}{*}{ E. cloacae (2) } & $S$ & $2(100)$ & - & $2(0.0)$ & $2(0.0)$ & $2(100)$ & $I(50.0)$ & $2(100)$ & $2(100)$ & $2(100)$ & $2(100)$ & - \\
\hline & 1 & - & - & - & - & - & $I(50.0)$ & - & - & - & - & $2(100)$ \\
\hline & $\mathrm{R}$ & - & $2(100)$ & - & - & - & - & - & - & - & - & - \\
\hline \multirow[t]{2}{*}{ K.oxytoca (I) } & $\mathrm{S}$ & $\mathrm{I}(0.0)$ & $\mathrm{I}(100)$ & - & & $I(100)$ & $I(100)$ & $\mathrm{I}(100)$ & $\mathrm{I}(100)$ & $\mathrm{I}(100)$ & - & - \\
\hline & $\mathrm{R}$ & - & - & $I(100)$ & $I(100)$ & & - & - & & & $I(100)$ & $I(100)$ \\
\hline \multirow[t]{2}{*}{ E. aerogens $(\mathrm{I})$} & $S$ & $I(100)$ & $I(100)$ & - & - & $I(100)$ & $\mathrm{I}(100)$ & $\mathrm{I}(100)$ & $\mathrm{I}(100)$ & I(100) & $\mathrm{I}(100)$ & $\mathrm{I}(100)$ \\
\hline & $\mathrm{R}$ & - & - & $I(100)$ & $\mathrm{I}(100)$ & - & - & - & - & - & - & - \\
\hline \multirow[t]{3}{*}{ Total (29) } & $S$ & $25(86.2)$ & $21(72.4)$ & I4(48.3) & $4(13.8)$ & $16(55.2)$ & $19(65.5)$ & $27(93.1)$ & $23(82.8)$ & $24(82.8)$ & $22(75.9)$ & $8(27.6)$ \\
\hline & I & $2(6.9)$ & I(3.4) & - & $2(6.9)$ & $2(6.9)$ & $2(6.9)$ & & $\mathrm{I}(3.4)$ & $\mathrm{I}(3.4)$ & $3(10.3)$ & $2(6.9$ \\
\hline & $R$ & $2(6.9)$ & $7(24.2)$ & $15(51.7)$ & $23(79.3)$ & II(37.9) & $8(27.6)$ & $2(6.9)$ & $5(17.3)$ & $4(13.8)$ & $4(13.8)$ & $19(65.5)$ \\
\hline
\end{tabular}

Abbreviations: GEN, gentamycin; CIP, ciprofloxacin; AMP, ampicillin; AMX, amoxicillin; AMC, amoxicillin clavulanic acid; TET, tetracycline; NIT, nitrofurantoin; CEF, ceftriaxone; CRX, cefuroxime; MER, meropenem; COT, co-trimoxazole; S, susceptible; I, intermediate; R, resistance; ASB, asymptomatic bacteriuria.

Table 5 Antimicrobial Susceptibility Profile of Gram-Positive Bacteria Isolated from Pregnant Women with ASB at Saint Paul's Hospital Millennium Medical College, Addis Ababa, Ethiopia, $2019(n=20)$

\begin{tabular}{|c|c|c|c|c|c|c|c|c|c|c|c|}
\hline \multicolumn{2}{|c|}{ Type of Isolates (n) } & \multicolumn{10}{|c|}{ Antimicrobial Agents n (\%) } \\
\hline & & GEN & ERY & CIP & PEN & СОT & TET & NIT & CD & AZT & AMP \\
\hline \multirow[t]{3}{*}{ S. aureus (10) } & $S$ & $9(90.0)$ & $6(60.0)$ & $9(90.0)$ & $3(30.0)$ & $7(70.0)$ & $7(70.0)$ & $10(100)$ & $8(80.0)$ & $9(90.0)$ & NA \\
\hline & I & $\mathrm{I}(10.0)$ & $\mathrm{I}(10.0)$ & $\mathrm{I}(10.0)$ & - & - & - & - & $\mathrm{I}(10.0)$ & - & NA \\
\hline & $\mathrm{R}$ & - & $3(30.0)$ & - & $7(70.0)$ & $3(30.0)$ & $3(30.0)$ & - & $I(10.0)$ & $\mathrm{I}(\mathrm{I0.0)}$ & NA \\
\hline \multirow[t]{3}{*}{ S. saprophyticus (8) } & $S$ & $6(75.0)$ & $4(50.0)$ & $6(75.0)$ & $2(25.0)$ & $4(50.0)$ & $4(50.0)$ & $8(100)$ & $6(75.0)$ & $7(87.5)$ & NA \\
\hline & I & $\mathrm{I}(25.0)$ & $2(25.0)$ & $\mathrm{I}(12.5)$ & - & $\mathrm{I}(12.5)$ & $2(25.0)$ & - & $\mathrm{I}(12.5)$ & $\mathrm{I}(12.5)$ & NA \\
\hline & $\mathrm{R}$ & $\mathrm{I}(25.0)$ & $2(25.0)$ & $\mathrm{I}(\mathrm{I} 2.5)$ & $6(75.0)$ & $3(37.5)$ & $2(25.0)$ & - & $\mathrm{I}(12.5)$ & - & NA \\
\hline \multirow[t]{3}{*}{ E. faecalis (2) } & $S$ & $2(100)$ & NA & $2(100)$ & $I(50.0)$ & $I(50.0)$ & $\mathrm{I}(50.0)$ & $2(100)$ & $2(100)$ & $2(100)$ & $I(50.0)$ \\
\hline & I & - & NA & - & - & - & $I(50.0)$ & - & - & - & - \\
\hline & $\mathrm{R}$ & - & NA & - & $I(50.0)$ & $I(50.0)$ & - & - & - & - & $I(50.0)$ \\
\hline \multirow[t]{3}{*}{ Total (20) } & $S$ & $17(85.0$ & $10(55.6)$ & $17(85.0)$ & $6(30.0)$ & $12(60.0)$ & $12(60.0)$ & $20(100)$ & $16(80.0)$ & $18(90.0$ & $I(50.0)$ \\
\hline & I & $2(10.0)$ & $3(16.7)$ & $2(10.0)$ & - & $I(5.0)$ & $3(I 5.0)$ & - & $2(10.0)$ & $I(5.0)$ & - \\
\hline & $\mathrm{R}$ & $I(5.0)$ & $5(27.7)$ & $I(5.0)$ & |4(70.0) & $7(35.0)$ & $5(25.0)$ & - & $2(10.0)$ & $I(5.0)$ & $I(50.0)$ \\
\hline
\end{tabular}

Abbreviations: GEN, gentamycin, ERY, erythromycin; CIP, ciprofloxacin; PEN, penicillin; COT, co-trimoxazole; TET, tetracycline; NIT, nitrofurantoin; CD, clindamycin; AZT, azithromycin; AMP, ampicillin; S, susceptible; I, intermediate; R, resistant; NA, not applicable; ASB, asymptomatic bacteriuria.

meropenem (75.2\%), and ciprofloxacin (75.2\%). This finding is comparable with a study conducted in Dessie, Ethiopia, ${ }^{5}$ Hawassa, Ethiopia, ${ }^{6}$ and Bale, Ethiopia. ${ }^{36}$ However, most Gram-negative bacteria were resistant to amoxicillin (79.3\%), amoxicillin-clavulanic acid (37.9\%).
The easy accessibility, indiscriminate and frequent use of antibiotics might cause the emergence of bacteria which are resistant to amoxicillin and amoxicillin-clavulanic acid.

Most E. coli isolated in this study were susceptible to gentamicin, nitrofurantoin, ceftriaxone, ciprofloxacin, and 
Table 6 Multi-Drug Resistance Profile of Bacteria Isolated from Pregnant Women with ASB at Saint Paul's Hospital Millennium Medical College, Addis Ababa, Ethiopia, $2019(n=28)$

\begin{tabular}{|c|c|c|}
\hline $\begin{array}{l}\text { Bacterial } \\
\text { Isolates }\end{array}$ & Combination of Antibiotics & $\mathbf{N}(\%)$ \\
\hline E. coli $(n=\mid 2)$ & $\begin{array}{l}\text { AMX, COT } \\
\text { AMX, MER } \\
\text { AMX, AMP, AMC, COT } \\
\text { AMX, AMP,AMC,TET,COT } \\
\text { AMX,AMP,AMC,CRT,CRX,COT } \\
\text { AMX,AMP,AMC,NIT,TET,COT } \\
\text { AMX,AMP,AMC, CTR,CRX,CRP,TET, } \\
\text { COT }\end{array}$ & $\begin{array}{l}\text { I }(8.3) \\
\text { I }(8.3) \\
3(25.0) \\
3(25.0) \\
\text { I }(8.3) \\
1(8.3) \\
2(16.7)\end{array}$ \\
\hline $\begin{array}{l}\text { K. pneumoniae } \\
(n=2)\end{array}$ & $\begin{array}{l}\text { AMX, CRP,MER,COT } \\
\text { AMX,AMP,AMC,CTR,CRX,GEN,CRP, } \\
\text { TET,MER,COT }\end{array}$ & $\begin{array}{l}\text { I }(50.0) \\
\text { I }(50.0)\end{array}$ \\
\hline K. oxytoca $(\mathrm{n}=\mathrm{I})$ & AMX,AMP,COT & $I(100)$ \\
\hline $\begin{array}{l}\text { Total (Gram- } \\
\text { negative) }\end{array}$ & & $15(5 \mid .7)$ \\
\hline S. aureus $(n=7)$ & $\begin{array}{l}\text { ERY,PEN } \\
\text { TET,PEN } \\
\text { COT,PEN } \\
\text { PEN,TET,CD,COT } \\
\text { PEN,TET,AZT,COT }\end{array}$ & $\begin{array}{l}3(42.9) \\
\mathrm{I}(14.2) \\
\mathrm{I}(14.2) \\
\mathrm{I}(14.2) \\
\mathrm{I}(14.2)\end{array}$ \\
\hline $\begin{array}{l}\text { S. saprophyticus } \\
(n=5)\end{array}$ & $\begin{array}{l}\text { PEN,CD } \\
\text { ERY,PEN } \\
\text { COT,ERY } \\
\text { GEN,PEN,TET,COT }\end{array}$ & $\begin{array}{l}I(20.0) \\
I(20.0) \\
I(20.0) \\
2(40.0)\end{array}$ \\
\hline $\begin{array}{l}\text { E. faecalis }(n=1) \\
\text { Total }(G r a m- \\
\text { positive }) \\
\text { Overall total }\end{array}$ & PEN, COT & $\begin{array}{l}1(100) \\
13(65) \\
28(57.1)\end{array}$ \\
\hline
\end{tabular}

Abbreviations: AMX, amoxicillin; COT, co-trimoxazole; CIP, ciprofloxacin; AMP, ampicillin; AMC, amoxicillin-clavulanic acid; TET, tetracycline; GEN, gentamycin; NIT, nitrofurantoin; CTR, ceftriaxone; CRX, cefuroxime; MER, meropenem; ERY, erythromycin; PEN, penicillin; CD, clindamycin; AZT, azithromycin; ASB, asymptomatic bacteriuria.

cefuroxime. However, most E. coli were resistant to ampicillin, cotrimoxazole, and amoxicillin-clavulanic acid. The proportion of antibiotic-resistant $E$. coli found in this study is higher than the proportion antibiotic-resistant $E$. coli reported from other parts of Ethiopia ${ }^{29}$ but it is low compared to report from Kenya. ${ }^{32}$ Moreover, the finding of this study is not in line with a report from India, where $6170 \%$ of $E$. coli were susceptible to ampicillin and amoxicillin-clavulanic acid, respectively. ${ }^{37}$ These differences could be due to variations in antibiotic prescription patterns across various countries.
All K. pneumoniae isolated in this study were susceptible to nitrofurantoin and most of them were susceptible to ampicillin, amoxicillin-clavulanic acid, and cefuroxime. The finding is not comparable with finding reported from Tanzania. ${ }^{31}$ All K. oxytoca and E. aerogens were resistant to ampicillin and amoxicillin. The high resistance to ampicillin and amoxicillin observed in this study may be due to the easy availability of antibiotics in local pharmacies and frequent prescription of antibiotics by physicians without antimicrobial susceptibility testing.

In the current study, none of the Gram-positive bacteria were resistant to nitrofurantoin. This could be due to the infrequent use of the drug in the study area. Most $S$. aureus isolated in the current study were resistant to penicillin, erythromycin, and tetracycline. This finding is not in line with the report from Togo. ${ }^{38}$ In addition, all E. faecalis were susceptible to gentamicin and ciprofloxacin; however, half of them were resistant to ampicillin and penicillin. In contrast, according to a study conducted in Togo, all E. faecalis were resistant to ciprofloxacin. ${ }^{38}$ In our study, a significant number $(57.1 \%)$ of bacteria were MDR which is lower than MDR reported from Jimma, Ethiopia (90\%), ${ }^{39}$ Dire Dawa, Ethiopia $(100 \%),{ }^{30}$ and Nigeria $(100 \%){ }^{40}$

\section{Limitation of the Study}

As participants may not remember all information asked during data collection, this study is prone to recall bias.

\section{Conclusion}

The prevalence of ASB among pregnant women in the present study was $16.9 \%$. History of UTI, history of catheterization, and history of natural abortion were significantly associated with ASB. The most prevalent bacteria isolated in this study were E. coli $(43.0 \%), S$. aureus (20.4\%), and S. saprophyticus (14.3\%). The majority of bacteria isolated in the present study were susceptible to nitrofurantoin and gentamicin. The study also showed that most of the bacteria were MDR.

\section{Abbreviations}

ANC, antenatal care; ASB, asymptomatic bacteriuria; ATCC, American Type Culture Collection; CFU, colonyforming unit; MDR, multidrug-resistance; HIV, human immunodeficiency virus; SPHMMC, Saint Paul's Hospital Millennium Medical College; UTI, urinary tract infection. 


\section{Data Sharing Statement}

All relevant data generated and analyzed are available within the paper.

\section{Ethical Approval and Consent to Participate}

The study was approved by the Institutional Review Board of Hawassa University, College of Medicine and Health Sciences (Ref No IRB/205/11) and Saint Paul's Hospital Millennium Medical College (Ref No pm23/368). An official permission letter was obtained from the Saint Paul's Hospital Millennium Medical College administration office. Participants enrolled in the study were informed about the study objectives, expected outcomes, benefits, and risks associated with it. Written informed consent was taken from all participants before the interview. The study was conducted in accordance with the Declaration of Helsinki.

\section{Acknowledgment}

We would like to thank Ethiopian Public Health Institutes Laboratory, Saint Paul's Hospital Millennium Medical College for facilitating the study. We also acknowledge all study participants for their willingness to take part in the study.

\section{Author Contributions}

All authors made a significant contribution to the work reported, whether that is in the conception, study design, execution, acquisition of data, analysis and interpretation, or in all these areas; took part in drafting, revising or critically reviewing the article; gave final approval of the version to be published; have agreed on the journal to which the article has been submitted; and agree to be accountable for all aspects of the work.

\section{Disclosure}

The authors declare no competing interests.

\section{References}

1. Onanuga A, Maureen CO, Deboh ED. Carriage of multi-drug resistant urobacteria by asymptomatic pregnant women in yenagoa, bayelsa state, Nigeria. Afr J Infect Dis. 2018;12(2):14-20. doi:10.21010/ajid. v12i 2.3

2. Radha S, Nambisan B, Prabhakaran NK, Jamal S. Prevalence and outcome of asymptomatic bacteriuria in early pregnancy. Int J Reproduction, Contraception, Obstet Gynecol. 2017;6 (1):223-227. doi:10.18203/2320-1770.ijrcog20164663

3. Aminu KY, Aliyu UU Asymptomatic bacteriuria in pregnant women in the antenatal booking clinic at aminu kano teaching hospital, Kano, Nigeria. Open J Obstet Gynecol. 2015; 5:286-297. doi:10.4236/ ojog.2015.55042
4. Tadesse S, Kahsay T, Adhanom G, et al. Prevalence, antimicrobial susceptibility profile and predictors of asymptomatic bacteriuria among pregnant women in Adigrat General Hospital, Northern Ethiopia. BMC Res Notes. 2018;11:740. doi:10.1186/s13104-0183911-7

5. Ali IE, Gebrecherkos T, Gizachew M, Menberu MA. Asymptomatic bacteriuria and antimicrobial susceptibility pattern of the isolates among pregnant women attending Dessie referral hospital, Northeast Ethiopia. Turk J Urol. 2018;44(3):251-260. doi:10.5152/ tud.2018.07741

6. Tessema NT, Ali MM, Zenebe MH. Bacterial associated urinary tract infection, risk factors, and drug susceptibility profile among adult people living with HIV at Hawassa University Comprehensive Specialized Hospital, Hawassa, Southern Ethiopia. Nature Scientific Rep. 2020;10:10790. doi:10.1038/s41598-020-67840-7

7. Al-Jendy AS, Al-Ofairi BA. Bacterial pathogens of patients with urinary tract infections and antibiotics susceptibility in Taiz Governorate-Yemen. Al-Mustansiriyah J Sci. 2019;30(1):43-51. doi:10.23851/mjs.v30i1.530

8. Labi AK, Yawson AE, Ganyaglo GY, Newman MJ. Prevalence and associated risk factors of asymptomatic bacteriuria in ante-natal clients in a large teaching hospital in ghana. Ghana Med J. 2015;49 (3):154-158. doi:10.4314/gmj.v49i3.5

9. Tadesse E, Teshome M, Merid Y, Kibret B, Shimelis T. Asymptomatic urinary tract infection among pregnant women attending the antenatal clinic of hawassa referral hospital, Southern Ethiopia. BMC Res Notes. 2014;7(155):1-5.

10. Elzayat MA, Ashton BV, Dabour ME, Cheng F. Prevalence of undiagnosed asymptomatic bacteriuria and associated risk factors during pregnancy: a cross-sectional study at two tertiary centres in Cairo, Egypt. BMJ Open. 2017;17(3):e013198.

11. Manjula R, Kavya H, Kashinakunti SV, Solabannavar S, Dorle AS, Lalitha HD. Diagnostic accuracy of griess test for asymptomatic bacteriuria in pregnancy. Br J Med Med Res. 2016;11(8):1-7. doi:10.9734/BJMMR/2016/20754

12. Paari P, Sindhuja TP, Dhinakaran S, Paul CM P. A cross-sectional study on asymptomatic bacteriuria among antenatal women attending an urban tertiary health care center in Southern India. Int $J$ Reprod Contracept Obstet Gynecol. 2017;6(10):4523. doi:10.18203/23201770.ijrcog20174435

13. Pantagada N, Kamala P, Asymptomatic bacteriuria among pregnant women attending a tertiary care Hospital. Int J Current Microbiol Applied Sci. 2017;6(3):523-529. doi:10.20546/ijcmas.2017.603. 061

14. Ramalingam K, Surasani VM, Bollu M. Prevalence of asymptomatic bacteriuria in antenatal women coming to NRIMC\&GH. Bangladesh J Obstet Gynaecol. 2015;30(1):30-36. doi:10.3329/bjog.v30i1.30505

15. Laily F, Lutan D, Amelia S, Tala MRZ, Nasution A T. Associated risk factors for urinary tract infection among pregnant women at Puskesmas Kenangan, Deli Serdang district. IOP Conf Series: Earth Environmental Sci. 2018;125:012035. doi:10.1088/1755-1315/ $125 / 1 / 012035$

16. Negussie A, Worku G, Beyene E. Bacterial identification and drug susceptibility pattern of urinary tract infection in pregnant Women at Karamara Hospital Jigjiga, Eastern Ethiopia. Afr J Bacteriol Res. 2018;10(2):15-22. doi:10.5897/JBR2017.0255

17. Ankur G, Namita S, Sapna G, et al. Prevalence of asymptomatic urinary tract infections in the three trimesters of pregnancy. Int J Curr Microbiol App Sci. 2015;(1):110-117.

18. Onyenweaku FC, Amah HC, Obeagu EI, Nwandikor UU, Onwuasoanya UF. Prevalence of asymptomatic bacteriuria and its antibiotic susceptibility pattern in pregnant women attending private ante natal clinics in Umuahia Metropolitan. Int J Curr Res Biol Med. 2017;2(2):13-23. doi:10.22192/ijcrbm.2017.02.02.00

19. Cheesbrough M. District Laboratory Practice in Tropical Countries, Part 2. New York: Cambridge University Press; 2006. 
20. CLSI. Performance Standards for Antimicrobial Susceptibility Testing. 29th ed ed. CLSI supplement M100 Wayne, PA: Clinical and Laboratory Standards Institiute; 2019.

21. Kamel HA, Hegab MH, Al-sehrawey AA, Hassan HM. Prevalence of asymptomatic bacteriuria in patients with preterm labor. Egyptian $J$ Hospital Med. 2018;73(9):7444-7447. doi:10.12816/ EJHM.2018.18699

22. Marami D, Balakrishnan S, Seyoum B. Prevalence, antimicrobial susceptibility pattern of bacterial isolates, and associated factors of urinary tract infections among HIV-positive patients at Hiwot Fana Specialized University Hospital, Eastern Ethiopia. Canadian J Infectious Diseases Med Microbiol. 2019;2019:1-8. doi:10.1155/ 2019/6780354

23. Khanum S, Ahmed JU, Khanam. Bacterial etiology, antibiotic sensitivity pattern and risk factors for asymptomatic bacteriuria during pregnancy: experience in a tertiary care Hospital. BIRDEM Med J. 2016;6(2):79-83. doi:10.3329/birdem.v6i2.31289

24. Nisha AK, Etana AE, Tesso H. Prevalence of asymptomatic bacteriuria during pregnancy in Adama city, Ethiopia. Int $J$ Microbiol Immunol Res. 20151;3(5):058-63.

25. Bayati AH, Ghaib AH, Nasraldeen N, Hamalaw SA, Zainab AH. Asymptomatic bacteriuria among pregnant women in sulaimani city. GJBB. 2016;5(3):327-330.

26. Tosin OA, Mathew AO, Bello FJ, Oludare IO, Dolapo AL, Abosede AF. Asymptomatic bacteriuria among antenatal women attending private hospital in lagos, Nigeria. Sch J App Med Sci. 2014;2(6D):3076-3080.

27. Abderrazzack AF, Aguid MN, Hig-Zounet BT, Tidjani A, Ameyapoh Y. Asymptomatic microbial urinary tract infection in HIV positive pregnant women in Ndjamena (Chad). Int J Innovations Biol Chem Sci. 2015;6:1-7.

28. Chukwu OS, Ezeonu IM, Victor MA, et al. Incidence, aetiology and antibiotic susceptibility profile of asymptomatic bacteriuria in pregnant women in Nsukka Urban, Enugu state, Nigeria. World J Life Sci Med Res. 2014;3(3):94.

29. Derbie A, Mekonnen D, Abate E, Tadesse S, Birku T, Biadglegne F. Bacterial isolates and their current drug susceptibility profile from urine among asymptomatic pregnant women attending at a referral hospital, northwest ethiopia. Ethiopian $J$ Reproductive Health. 2018;10(2):1-10.

30. Alemu A, Moges F, Shiferaw Y, et al. Bacterial profile and drug susceptibility pattern of urinary tract infection in pregnant women at University of Gondar Teaching Hospital, Northwest Ethiopia. BMC Res Notes. 2012;5(197):1-7.
31. Mwei MK, Mchome B, John B, Maro B. Asymptomatic bacteriuria among pregnant women attending antenatal clinic at kilimanjaro christian medical centre in Northern Tanzania. Clin Pract. 2018;15 (6):917-922.

32. Ayoyi AO, Kikuvi G, Bii C, Kariuki S. Prevalence, aetiology and antibiotic sensitivity profile of asymptomatic bacteriuria isolates from pregnant women in selected antenatal clinic from Nairobi, Kenya. Pan Afr Med J. 2017;26:1-12. doi:10.11604/pamj.2017.26.41.10975

33. Mwambete KD, Msigwa M. Prevalence of asymptomatic urinary tract infections among pregnant women residing in a rural and an urban area in Tanzania. East Central African J Pharm Sci. 2017;20 (13):27-32.

34. Mohamed NR, Omar H, Abd-Allah IM. Prevalence and risk factors of urinary tract infection among pregnant women in Ismailia City, Egypt. IOSR J Nursing Health Sci. 2017;19592320. doi:10.9790/ 1959-0603076272

35. Nguefack CT, Ebongue CO, Chokotheu CN, Ewougo CE, Njamen TN. Clinical presentation, risk factors and pathogens involved in bacteriuria of pregnant women attending antenatal clinic of 3 hospitals in a developing country: a cross sectional analytic study. BMC Pregnancy Childbirth. 2019;19(143):1-6.

36. Taye S, Getachew M, Desalegn Z, Biratu A, Mubashir K, Bacterial profile, antibiotic susceptibility pattern and associated factors among pregnant women with urinary tract infection in Goba and Sinana Woredas, Bale Zone, Southeast Ethiopia. BMC Res Notes. 2018;11 (799):799. doi:10.1186/s13104-018-3910-8

37. Sujatha R, Nawani M. Prevalence of asymptomatic bacteriuria and its antibacterial susceptibility pattern among pregnant women attending the antenatal clinic at Kanpur, India. J Clin Diagnostic Res. 2014;8 (4):C01C03. doi:10.7860/JCDR/2014/6599.4205

38. Foudaa AA, Seglab TD, Mounerouc S, et al. Sensibility of uropathogens in pregnant women with asymptomatic bacteriuria in Lome, Togo. Scientific J Biol Sci. 2015;4(4):30-35. doi:10.14196/sjbs. v4i4.1866

39. Gizachew Z, Kassa T, Beyene G, Howe R, Yeshitila B. Multi-Drug resistant bacteria and associated factors among reproductive age women with significant bacteriuria. Ethiop Med J. 2019;1:31-43.

40. Akpan NG, Umoyen AJ, Luka TT, Onwuezobe IA, Antia UE, Okon AS. Asymptomatic uropathogenic bacteriuria among pregnant and non-pregnant women at St Luke's Hospital Anua, Offot Ukwa District Uyo: A reassessment case-control approach. American J Lab Med. 2019;4(1):1-10. doi:10.11648/j.ajlm.20190401.11
Therapeutics and Clinical Risk Management

\section{Publish your work in this journal}

Therapeutics and Clinical Risk Management is an international, peerreviewed journal of clinical therapeutics and risk management, focusing on concise rapid reporting of clinical studies in all therapeutic areas, outcomes, safety, and programs for the effective, safe, and sustained use of medicines. This journal is indexed on PubMed Central, CAS,
EMBase, Scopus and the Elsevier Bibliographic databases. The manuscript management system is completely online and includes a very quick and fair peer-review system, which is all easy to use. Visit http://www.dovepress.com/testimonials.php to read real quotes from published authors. 